\title{
Fluctuations of pion elliptic flow, triangular flow, and HBT correlation functions in ultrarelativistic heavy ion collisions
}

\author{
Ying $\mathrm{Hu}^{1}$, Wei-Ning Zhang ${ }^{1,2 *}$, Yan-Yu Ren ${ }^{2}$ \\ ${ }^{1}$ School of Physics and Optoelectronic Technology, \\ Dalian University of Technology, Dalian, Liaoning 116024, China \\ ${ }^{2}$ Department of Physics, Harbin Institute of Technology, \\ Harbin, Heilongjiang 150006, China
}

\begin{abstract}
We investigate the fluctuations of pion elliptic flow, triangular flow, and Hanbury-Brown-Twiss (HBT) correlation functions for the hydrodynamic sources with fluctuating initial conditions in the heavy ion collisions of the $\mathrm{Au}-\mathrm{Au}$ at $\sqrt{s_{N N}}=200 \mathrm{GeV}$ and the $\mathrm{Pb}-\mathrm{Pb}$ at $\sqrt{s_{N N}}=2.76 \mathrm{TeV}$. A method based on event-subcollection analysis is used to detect these fluctuations in ultrarelativistic heavy ion collisions. We introduce a granularity length to describe the granular inhomogeneity of the initial sources, and investigate its relationships with the fluctuations of the flow harmonics and HBT correlation functions. Our investigations indicate that the fluctuations of the triangular flow of event subcollections are sensitive to the granularity length of the initial source. This dependence provide a way to investigate the granular inhomogeneity of the initial source through analysing the fluctuations of triangular flow in ultrarelativistic heavy ion collisions.
\end{abstract}

PACS numbers: 25.75.-q, 25.75.Gz, 25.75.Ld

\footnotetext{
*Email: wnzhang@dlut.edu.cn
} 


\section{INTRODUCTION}

The primary goal of the heavy ion collisions at the highest energy of the Relativistic Heavy Ion Collider (RHIC) and the energy of the Large Hadron Collider (LHC) is to explore the properties of the extreme hot and dense matter, the quark-gluon plasma (QGP), existed in the early stages of the collisions. Recent investigations [1, 2] indicate that the initial systems created in the ultrarelativistic heavy ion collisions at the RHIC and LHC energies are not uniform in space, and there are event-by-event fluctuations of the system initial quantities, due to the fluctuations of nucleon distributions in the nuclei, the fluctuations in the color charge distributions inside a nucleon, and combined with highly Lorentz contraction. The studies of the system evolution with the fluctuating initial conditions (FIC) and the influence of the FIC on final particle observables are recently very interesting issues in high energy heavy ion collisions [1]. They are important to improve our understanding of the experimental results at the RICH and the LHC.

Elliptic flow and two-particle Hanbury-Brown-Twiss (HBT) correlation functions are important observables in high energy heavy ion collisions. They reflect the transverse (perpendicular to the beam direction) anisotropic pressure property and the space-time structure of the particle-emitting sources, respectively. In ultrarelativistic heavy ion collisions, the spectators depart from the reaction region quickly after collision, and a very hot and dense fireball is created in the mid-rapidity region. For uniform systems of the fireballs the oddorder azimuthal flow harmonics are expected to be zero. However, recent studies indicate that the fluctuating inhomogeneous density distributions of the initial systems may lead to nonzero triangular flow, and thus inspires the investigations of azimuthal triangular flow and even higher-order flow harmonics [1 11]. In Ref. [8], the authors reproduced well the experimental flow results of $v_{2}, v_{3}, v_{3}$, and $v_{5}$ in the ultrarelativistic heavy ion collisions at the RHIC and the LHC, by using the IP-Glasma FIC [2] with the viscous hydrodynamic model of MUSIC [6]. The investigations imply that the fluctuations in the initial geometry state are important and the created medium behaves as a nearly perfect liquid of nuclear matter because it has an extraordinarily low ratio of shear viscosity to entropy density.

On event-by-event basis, the particle-emitting sources with the FIC are bumpy and inhomogeneous [6, 12 16]. This inhomogeneous structure may lead to the fluctuations of final observables event-by-event. Although the influence of the IFC can be analysed by some 
observables, for instance, nonzero $v_{3}$, our motivation here is to detect the fluctuations of final observables directly and try to look for the relationship between the fluctuations of final observables and the granular inhomogeneity of the initial sources.

In this paper, we use the Heavy Ion Jet Interaction Generator (HIJING) [17] to generate the FIC of the particle-emitting sources for the heavy ion collisions of the $\mathrm{Au}-\mathrm{Au}$ at $\sqrt{s_{N N}}=$ $200 \mathrm{GeV}$ at the RHIC and the $\mathrm{Pb}-\mathrm{Pb}$ at $\sqrt{s_{N N}}=2.76 \mathrm{TeV}$ at the LHC. The system evolution is described by the relativistic ideal hydrodynamics in $(2+1)$ dimensions with the Bjorken longitudinal boost invariance [18], and with the equation of state (EOS) of s95pPCE [19]. We use the relativistic Harten-Lax-Leer-Einfeldt (RHLLE) algorithm [20-25] in our hydrodynamic calculations. Although this algorithm is also valid for the viscous hydrodynamic source with rest and smoothed initial conditions [26], it is hard to obtain the stable numerical solutions for the viscous hydrodynamics with the FIC and nonzero initial velocities of fluid-cells in the source with the RHLLE algorithm. We calculate the pion elliptic flow, triangular flow, and HBT correlation functions for the ideal hydrodynamic sources with the FIC and the nonzero initial fluid velocity. Motivated by the works on the fluctuations of single-event HBT correlation functions for granular sources [14, 16, 27, 28], we investigate the fluctuations of the flow harmonics and HBT correlation functions of event subcollections for the FIC sources. We introduce a granularity length to describe the granular inhomogeneity of the initial sources and investigate its relationships with the fluctuations of the flow harmonics and HBT correlation functions. Our investigations indicate that the FIC lead to event-by-event fluctuations of the elliptic flow, triangular flow, and HBT correlation functions. These FIC-caused fluctuations can be detected by the fluctuation distributions of the observables of event subcollections. The fluctuations of the triangular flow of event subcollections are sensitive to the granularity length of the initial source. This dependence provide a way to investigate the granular inhomogeneity of the initial sources through analysing the fluctuations of triangular flow in ultrarelativistic heavy ion collisions.

The rest of the paper is organized as follows. In Sec. II, we will give a description on solving the hydrodynamic equations in $(2+1)$ dimensions and present the space-time evolutions of the sources with the FIC. In Sec. III, we will investigate the fluctuations of the pion elliptic flow, triangular flow, and HBT correlation functions in the event subcollections for the heavy ion collisions of the Au-Au collisions at $\sqrt{s_{N N}}=200 \mathrm{GeV}$ at the RHIC and the $\mathrm{Pb}-\mathrm{Pb}$ at $\sqrt{s_{N N}}=2.76 \mathrm{TeV}$ at the LHC. The relationship between the fluctuations of 
the observables of event subcollections and the granular inhomogeneity of initial source is investigated also in Sec. III. Finally, we will give the summary and discussions in Sec. IV.

\section{HYDRODYNAMIC EVOLUTION OF THE SOURCES WITH HIJING FIC}

The description of ideal hydrodynamics for the system with zero net-baryon density is defined by the local conservations of energy and momentum[20, 29],

$$
\partial_{\mu} T^{\mu \nu}=0
$$

where $T^{\mu \nu}=(\epsilon+\mathcal{P}) u^{\mu} u^{\nu}-\mathcal{P} g^{\mu \nu}$ is the density tensor of energy-momentum of ideal fluid, $\epsilon$ and $\mathcal{P}$ are the energy density and pressure in the local rest frame of the fluid element which moving with velocity $\boldsymbol{v}, u^{\mu}=\gamma(1, \boldsymbol{v})$ is the four-velocity, $\gamma=\left(1-\boldsymbol{v}^{2}\right)^{-1 / 2}$, and $g^{\mu \nu}=$ $\operatorname{diag}(+,-,-,-)$ is Minkowski metric tensor. Under the assumption of Bjorken longitudinal boost invariance [18], the hydrodynamics in $(3+1)$ dimensions reduces to $(2+1)$ dimensions. In this case we need only to solve the transverse equations of motion in $z=0$ plane, and the hydrodynamic solutions at $z \neq 0\left(v^{z}=z / t\right)$ can be obtained by the longitudinal boost invariance hypothesis [12, 30].

From Eq. (1) we have the transverse equations in $z=0$ plane,

$$
\begin{aligned}
\partial_{t} \mathcal{E}+\partial_{x}\left[(\mathcal{E}+\mathcal{P}) v^{x}\right]+\partial_{y}\left[(\mathcal{E}+\mathcal{P}) v^{y}\right] & =-\mathcal{F}(\mathcal{E}, \mathcal{P}, t), \\
\partial_{t} \mathcal{M}^{x}+\partial_{x}\left(\mathcal{M}^{x} v^{x}+\mathcal{P}\right)+\partial_{y}\left(\mathcal{M}^{x} v^{y}\right) & =-\mathcal{G}\left(\mathcal{M}^{x}, t\right), \\
\partial_{t} \mathcal{M}^{y}+\partial_{x}\left(\mathcal{M}^{y} v^{x}\right)+\partial_{y}\left(\mathcal{M}^{y} v^{y}+\mathcal{P}\right) & =-\mathcal{G}\left(\mathcal{M}^{y}, t\right),
\end{aligned}
$$

where $\mathcal{E}=T^{00}, \mathcal{M}^{i}=T^{0 i},(i=x, y), \mathcal{F}(\mathcal{E}, \mathcal{P}, t)=(\mathcal{E}+\mathcal{P}) / t$, and $\mathcal{G}\left(\mathcal{M}^{i}, t\right)=\mathcal{M}^{i} / t$. In equation set (2) there are $\epsilon, \mathcal{P}, v^{x}$, and $v^{y}$ four variables. So an EOS, $\mathcal{P}(\epsilon)$, is needed to enclose the equation set. In the calculations, we use the EOS of s95p-PCE, which combines the hadron resonance gas at low temperature and the lattice QCD results at high temperature [19].

Assuming the local equilibrium of system is reached at time $\tau_{0}$, we construct the initial energy density of the hydrodynamic source at $z=0$, by using the AMPT code [31] in which the HIJING is used for generating the initial conditions, as [12, 32$]$

$$
\epsilon\left(\tau_{0}, x, y ; z=0\right)=K \sum_{\alpha} \frac{p_{\perp \alpha}}{\tau_{0}} \frac{1}{2 \pi \sigma_{0}^{2}} \exp \left\{-\frac{\left[x-x_{\alpha}\left(\tau_{0}\right)\right]^{2}+\left[y-y_{\alpha}\left(\tau_{0}\right)\right]^{2}}{2 \pi \sigma_{0}^{2}}\right\} .
$$


Here $p_{\perp \alpha}$ is the transverse momentum of parton $\alpha$ in the fluid element at $(x, y), x_{\alpha}\left(\tau_{0}\right)$ and $y_{\alpha}\left(\tau_{0}\right)$ are the transverse coordinates of the parton at $\tau_{0}, \sigma_{0}$ is a transverse width parameter, and $K$ is a scale factor which can be adjusted to fit the experimental data of produced hadrons [32]. The initial velocity of the fluid element is then determined by the initial energy density and the average transverse momentum of the partons in the element.

With the EOS and the initial values of energy density and velocities, we can solve equation set (2) using the relativistic HLLE scheme and Sod's operation splitting method [20 25, 33]: first getting the solutions for the corresponding homogeneous equations in $x$ and $y$ directions; then obtaining the solutions of (2) with the corrections of $\mathcal{F}$ and $\mathcal{G}$ to the solutions of the homogeneous equations. In our calculations the spatial grid sizes are taken to be $\Delta x=\Delta y=0.1 \mathrm{fm}$, and the time step is taken to be $\Delta t=0.99 \Delta x[22-25]$.

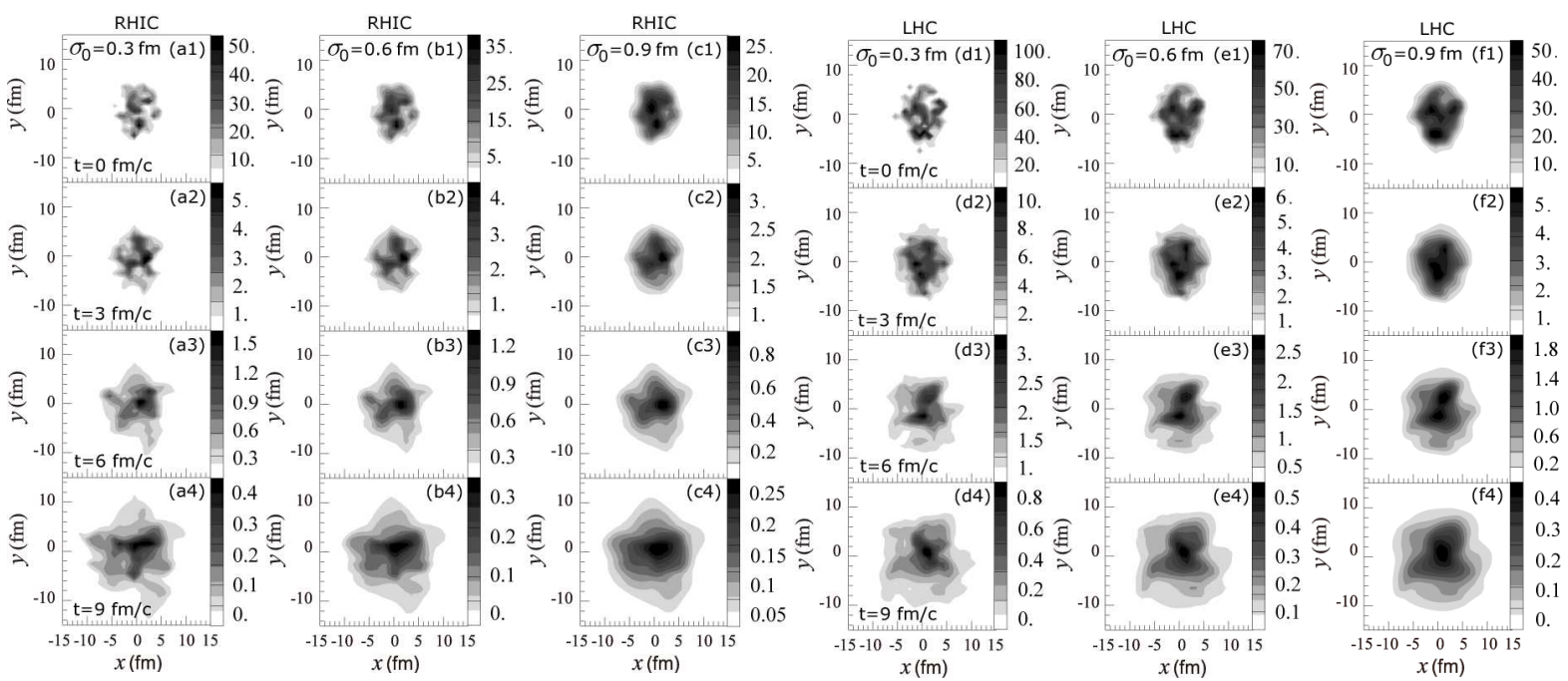

FIG. 1: The transverse distributions of energy density at $z=0$ for the Au-Au collisions at $\sqrt{s_{N N}}=$ $200 \mathrm{GeV}$ at the RHIC and the $\mathrm{Pb}-\mathrm{Pb}$ collisions at $\sqrt{s_{N N}}=2.76 \mathrm{TeV}$ at the LHC. The impact parameter $b$ for both the RHIC and LHC heavy ion collisions is $4 \mathrm{fm}$. The $\sigma_{0}$ values are $0.3,0.6$, and $0.9 \mathrm{fm}$. The panels [(a1)-(f1)], [(a2)-(f2)], [(a3)-(f3)], [(a4)-(f4)] are for the evolution time $t=0,3,6$, and $9 \mathrm{fm} / c$ after $\tau_{0}$, respectively. The unit of energy density is $\mathrm{GeV} / \mathrm{fm}^{3}$.

In Figs. 1 $(\mathrm{a} 1)-(\mathrm{c} 1)]$ and 1[(d1)- (f1)], we show the transverse distributions of the source initial energy density at $z=0$ for the $\mathrm{Au}-\mathrm{Au}$ and $\mathrm{Pb}-\mathrm{Pb}$ collisions at the RHIC and LHC energies, respectively. Here the unit of energy density is $\mathrm{GeV} / \mathrm{fm}^{3}$. The impact parameter $b$ for both the RHIC and LHC heavy ion collisions is $4 \mathrm{fm}$, and the $\tau_{0}$ values for the RHIC and LHC collisions are taken to be 0.6 and $0.4 \mathrm{fm} / c$, respectively. The energy densities at the 
evolution time 3, 6, and $9 \mathrm{fm} / c$ after $\tau_{0}$ are shown in the panels [(a2)- (a4)], [(b2)-(b4)], $[(\mathrm{c} 2)-(\mathrm{c} 4)],[(\mathrm{d} 2)-(\mathrm{d} 4)],[(\mathrm{e} 2)-(\mathrm{e} 4)]$, and [(f2)-(f4)] for the RHIC and LHC sources with the different $\sigma_{0}$, respectively. One can see from the panels $[(\mathrm{a} 1)-(\mathrm{c} 1)]$ and $[(\mathrm{d} 1)-(\mathrm{f} 1)]$ that the initial energy density is fluctuated. There are hot spots and cold valleys in the systems. We call this inhomogeneous structure the granular inhomogeneity of the initial source. The maximum of the energy density of spot decreases when $\sigma_{0}$ increases. Also, the spot number decreases with increasing $\sigma_{0}$. From the panels of time greater than zero one can see that the sources are still inhomogeneous at the late stages of the evolution, due to the initial fluctuations.
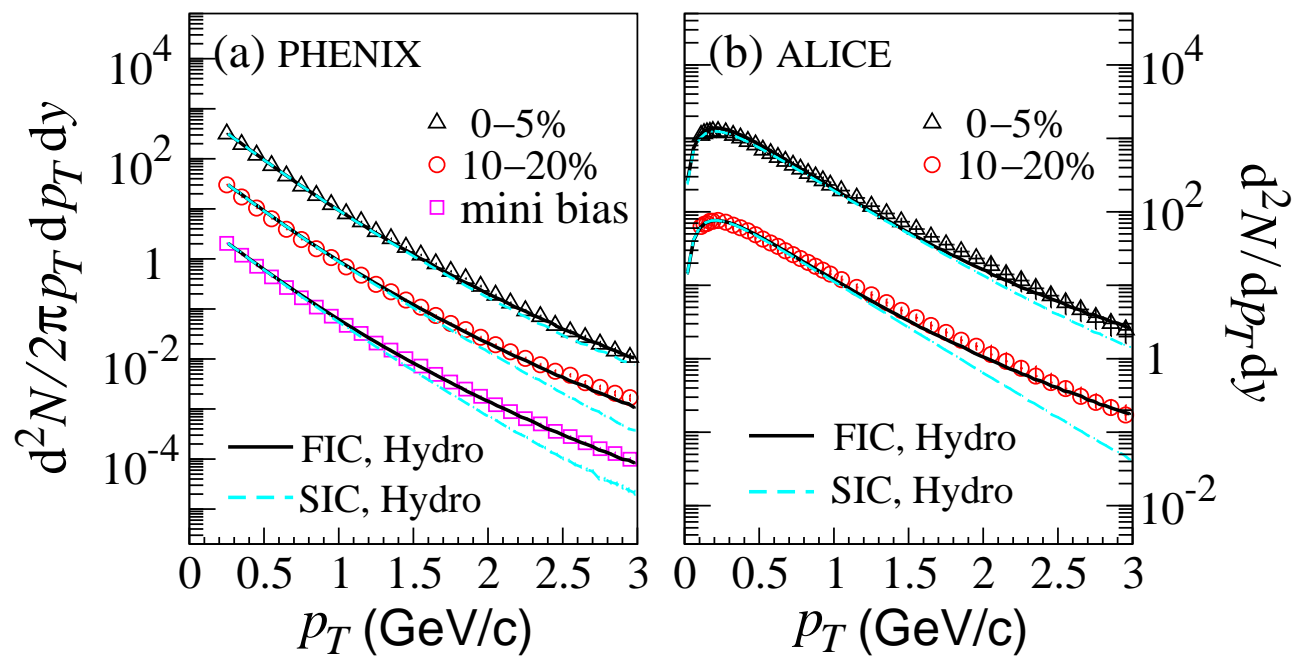

FIG. 2: (Color online) The pion transverse momentum spectra for the Au-Au collisions at the RHIC energy $\sqrt{s_{N N}}=200 \mathrm{GeV}$ [panel (a)] and the Pb-Pb collisions at the LHC energy $\sqrt{s_{N N}}=2.76$ $\mathrm{TeV}$ [panel(b)]. The solid lines are the hydrodynamic results with the FIC, and the dashed lines are the hydrodynamic results with the SIC which are obtained by averaging the FIC over 100 events. The circle, triangle, and square symbols are the experimental data at the RHIC [34] and the LHC [35].

We show in Figs. 2(a) and 2(b) the pion transverse momentum spectra calculated by the hydrodynamics with the FIC (solid lines) and the smoothed initial conditions (SIC) which are obtained by averaging the FIC over 100 events, for the $\mathrm{Au}-\mathrm{Au}$ and $\mathrm{Pb}-\mathrm{Pb}$ collisions at the RHIC and LHC energies, respectively. Here, the circle, triangle, and square symbols are the experimental data at the RHIC [34] and the LHC [35]. In the hydrodynamic calculations, we take the particle rapidity cuts the same as in the experimental analyses at the RHIC [34] 
and the LHC [35], respectively. The freeze out temperature is taken to be $130 \mathrm{MeV}$, and the parameter $\sigma_{0}$ is $0.6 \mathrm{fm}$. For the centralities $0-5 \%, 10-20 \%$, and mini bias, the regions of impact parameter are taken to be $0-2.3,4.2-5.9$, and $0-10.2 \mathrm{fm}$, respectively [36]. It can be seen that the hydrodynamic results with the FIC are consistent with the experimental data. At large $p_{T}$, the spectrum of the hydrodynamic source with the FIC is higher than the corresponding spectrum of the hydrodynamic source with the SIC.

\section{FLUCTUATIONS OF PION FLOW HARMONICS AND HBT CORRELA- TION FUNCTIONS}

\section{A. Flow harmonics of event subcollections}

In high energy heavy ion collisions, the invariant momentum distribution of final particles can be written in the form of a Fourier series [37, 38],

$$
E \frac{d^{3} N}{d^{3} p}=\frac{1}{2 \pi} \frac{d^{2} N}{p_{T} d p_{T} d y}\left[1+\sum_{n} 2 v_{n} \cos \left(n \phi-n \Psi_{R}\right)\right],
$$

where $\phi$ is the azimuthal angle of the particle and $\Psi_{R}$ is the azimuthal angle of event reaction plane. The first term on the right side of Eq. (44) is the transverse momentum spectrum, and the coefficients in the summation, $v_{n}=\left\langle\cos \left[n\left(\phi-\Psi_{R}\right)\right]\right\rangle$, are the azimuthal $n$ th-order flow harmonics, where $\langle\ldots\rangle$ denotes the average over the particles and events. In experimental data analyses, the reaction plane is usually replaced by the event plane which is determined with the measured particles in an event [39, 40]. An alternative technique in flow analyses is the measurement of the two-particle cumulant of azimuthal correlations, $\left[v_{n}\{2\}\right]^{2}=\left\langle\cos \left[n\left(\phi_{1}-\phi_{2}\right)\right]\right\rangle[41,42]$, which avoids the uncertainty in estimating reaction plane. In this work, we calculate the integrated flow harmonics $v_{n}^{\prime}$ and $p_{T}$-differential flow harmonics $v_{n}\left(p_{T}\right)$ with the two-particle cumulant method [41] as,

$$
\begin{gathered}
v_{n}^{\prime}\{2\}=\left[\frac{N_{n}\{2\}}{N_{\text {pair }}}\right]^{\frac{1}{2}}=\left\{\frac{1}{N_{\text {pair }}} \sum_{\alpha=1}^{N_{\text {evt }}} \sum_{i \neq j}^{M} \cos n\left(\phi_{i}-\phi_{j}\right)\right\}^{\frac{1}{2}}, \\
v_{n}\{2\}\left(p_{T}\right)=\frac{N_{n}\{2\}\left(p_{T}\right)}{N_{\text {pair }}\left(p_{T}\right)} \frac{1}{v_{n}^{\prime}\{2\}},
\end{gathered}
$$

where $N_{\text {pair }}=N_{\text {evt }} M(M-1) / 2$ is the total number of the particle pairs in $N_{\text {evt }}$ events, $M$ is the particle multiplicity of event, $N_{\text {pair }}\left(p_{T}\right)$ and $N_{n}\{2\}\left(p_{T}\right)$ are the counts of the particle pairs in the $p_{T}$ bin with the weights 1 and $\cos \left[n\left(\phi_{i}-\phi_{j}\right)\right]$, respectively. 


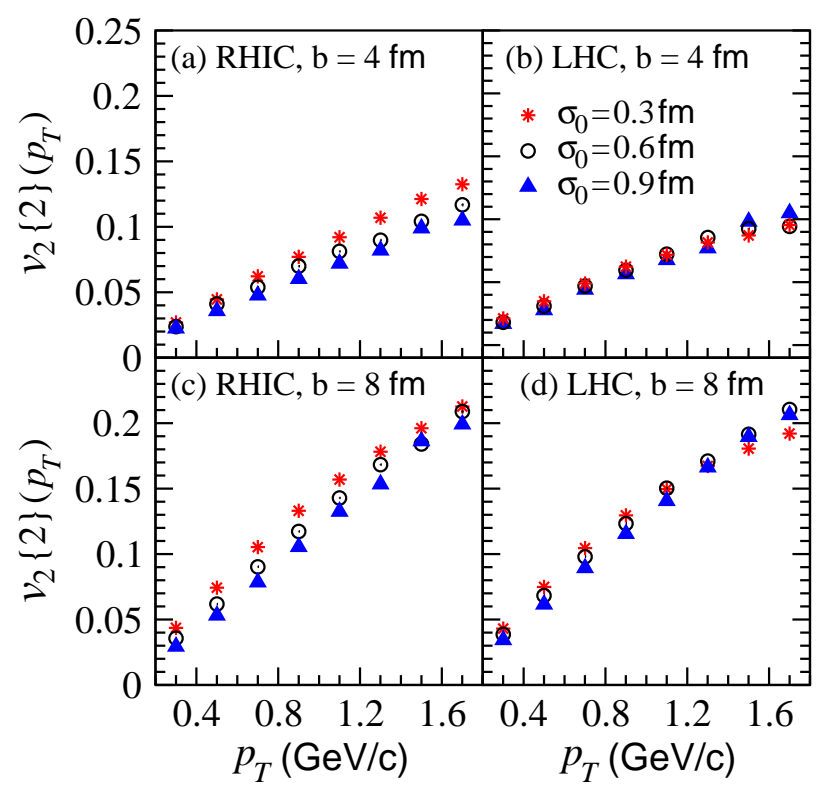

FIG. 3: (Color online) The pion elliptic flow of the hydrodynamic sources for the Au-Au collisions at the RHIC energy $\sqrt{s_{N N}}=200 \mathrm{GeV}$ and the $\mathrm{Pb}-\mathrm{Pb}$ collisions at the LHC energy $\sqrt{s_{N N}}=2.76$ TeV with impact parameters $b=4$ and $8 \mathrm{fm}$. The star, circle, and triangle symbols are for the parameter $\sigma_{0}=0.3,0.6$ and $0.9 \mathrm{fm}$, respectively.

In Fig. 3 we plot the pion $p_{T}$-differential elliptic flow of the hydrodynamic sources for the $\mathrm{Au}-\mathrm{Au}$ collisions at the RHIC energy $\sqrt{s_{N N}}=200 \mathrm{GeV}$ and the $\mathrm{Pb}-\mathrm{Pb}$ collisions at the LHC energy $\sqrt{s_{N N}}=2.76 \mathrm{TeV}$ with impact parameters $b=4$ and $8 \mathrm{fm}$. In the calculations, the number of events $N_{\text {evt }}$ is six thousand and the particle-pair number for each event is taken to be $10^{6}$. One can see that the values of elliptic flow decrease with increasing $\sigma_{0}$ for the RHIC sources, and are almost independent of $\sigma_{0}$ for the LHC sources.

For the hydrodynamic sources with the FIC, the elliptic flow and triangular flow of single event vary event to event greatly. These event-by-event fluctuations are associated with the FIC as well as the sample statistics. To reduce the effect of sample statistics on the fluctuations, we will examine the elliptic flow and triangular flow of the event subcollections, each of them has enough events. In Fig. 4 the dashed lines show the pion elliptic flow of the event subcollections each of them with 100 events, for the RHIC and the LHC sources with different impact parameter $b$ and $\sigma_{0}$ values. The number of particle pair for each event is $10^{6}$. The solid lines are the results averaged over sixty the subcollections. The error bars of the flow harmonics of the event subcollections are calculated based on the definition (6) 


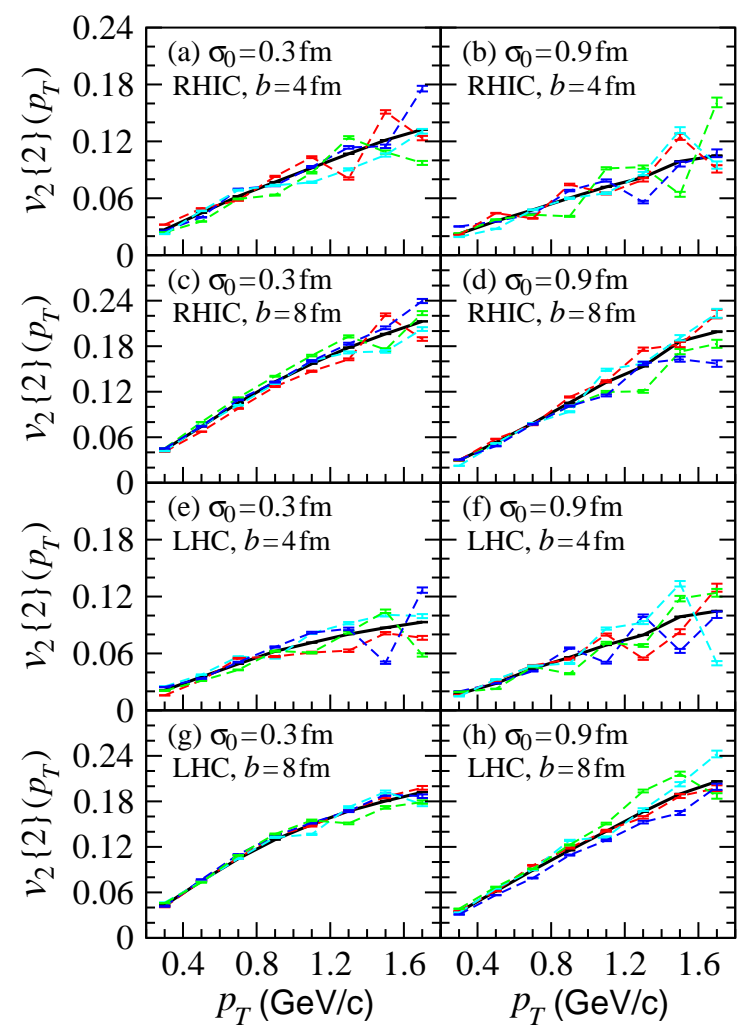

FIG. 4: (Color online) The pion elliptic flow of the event subcollections (dashed lines) each of them with 100 events, for the RHIC and the LHC sources with $b=4$ and $8 \mathrm{fm}, \sigma_{0}=0.3,0.6$, and 0.9 $\mathrm{fm}$. The number of particle pair for each event is $10^{6}$. The solid lines are the average results over sixty the subcollections.

and the statistical counts, by

$$
\begin{aligned}
\Delta & {\left[v_{n}\{2\}\left(p_{T}\right)\right]=\Delta\left[\frac{N_{n}\{2\}\left(p_{T}\right)}{N_{\text {pair }}\left(p_{T}\right)}\right] \frac{1}{v_{n}^{\prime}\{2\}}+\frac{N_{n}\{2\}\left(p_{T}\right)}{N_{\text {pair }}\left(p_{T}\right)} \Delta\left[\frac{1}{v_{n}^{\prime}\{2\}}\right] } \\
& =v_{n}\{2\}\left(p_{T}\right)\left\{\frac{\Delta\left[N_{n}\{2\}\left(p_{T}\right)\right]}{N_{n}\{2\}\left(p_{T}\right)}+\frac{\Delta\left[N_{\text {pair }}\left(p_{T}\right)\right]}{N_{\text {pair }}\left(p_{T}\right)}+\frac{\Delta\left[N_{n}\{2\}\right]}{2 N_{n}\{2\}}+\frac{\Delta N_{\text {pair }}}{2 N_{\text {pair }}}\right\} \\
& =v_{n}\{2\}\left(p_{T}\right)\left[\frac{1}{\sqrt{N_{n}\{2\}\left(p_{T}\right)}}+\frac{1}{\sqrt{N_{\text {pair }}\left(p_{T}\right)}}+\frac{1}{2 \sqrt{N_{n}\{2\}}}+\frac{1}{2 \sqrt{N_{\text {pair }}}}\right] \\
& \approx v_{n}\{2\}\left(p_{T}\right)\left[\frac{1}{\sqrt{N_{n}\{2\}\left(p_{T}\right)}}+\frac{1}{\sqrt{N_{\text {pair }}\left(p_{T}\right)}}\right] .
\end{aligned}
$$

One can see from Fig. 4 that the elliptic flow calculated with the 100 events are still with great fluctuations. They are larger than the statistical errors. So, the fluctuations of the flow of event subcollections reflect the intrinsic properties of the sources. 
The azimuthal flow harmonics are related to the initial eccentricities of the source [3, 9$]$,

$$
\varepsilon_{n}=\frac{\sqrt{\left\{\rho^{2} \cos \left(n \phi^{\prime}\right)\right\}^{2}+\left\{\rho^{2} \sin \left(n \phi^{\prime}\right)\right\}^{2}}}{\left\{\rho^{2}\right\}},
$$

where $\phi^{\prime}$ is the azimuthal angle related to the reaction plane ( $x z$ plane), $\boldsymbol{\rho}=(x, y)$ is the transverse coordinate of initial source point, and $\{\cdots\}$ denotes the average over initial source. In order to give an approximate estimation of the elliptic flow for the FIC sources, we use a simple distribution of the initial source with separated droplets to calculate the eccentricity $\varepsilon_{2}$. The single-event transverse distribution of the initial granular source is given by [27, 43]

$$
D(\boldsymbol{\rho})=\frac{1}{N_{d}\left(2 \pi a^{2}\right)} \sum_{i=1}^{N_{d}} \exp \left[-\frac{\left(\boldsymbol{\rho}-\boldsymbol{R}_{\perp i}\right)^{2}}{2 a^{2}}\right],
$$

where $N_{d}$ is the number of droplet, $\boldsymbol{R}_{\perp i}=\left(X_{i}, Y_{i}\right)$ are the transverse coordinates of droplet centers, and $a$ is the standard deviation of the Gaussian distribution. So, for a single event we have

$$
\begin{gathered}
\left\{\rho^{2} \cos \left(2 \phi^{\prime}\right)\right\}=\frac{1}{N_{d}} \sum_{i=1}^{N_{d}}\left(Y_{i}^{2}-X_{i}^{2}\right), \quad\left\{\rho^{2} \sin \left(2 \phi^{\prime}\right)\right\}=\frac{1}{N_{d}} \sum_{i=1}^{N_{d}} 2 X_{i} Y_{i}, \\
\left\{\rho^{2}\right\}=\frac{1}{N_{d}} \sum_{i=1}^{N_{d}}\left(2 a^{2}+X_{i}^{2}+Y_{i}^{2}\right) .
\end{gathered}
$$

Further, assume that the central coordinates of the droplets obey the Gaussian distribution, $P\left(X_{i}, Y_{i}\right) \sim \exp \left(-X_{i}^{2} / 2 \mathcal{R}_{x}^{2}-Y_{i}^{2} / 2 \mathcal{R}_{y}^{2}\right)$. By substituting the summation $\frac{1}{N_{d}} \sum_{i}$ in Eqs. (10) and (11) with $\int d X_{i} d Y_{i} P\left(X_{i}, Y_{i}\right)$ and completing the integrations, we get

$$
\left\{\rho^{2} \cos \left(2 \phi^{\prime}\right)\right\}=\mathcal{R}_{y}^{2}-\mathcal{R}_{x}^{2}, \quad\left\{\rho^{2} \sin \left(2 \phi^{\prime}\right)\right\}=0, \quad\left\{\rho^{2}\right\}=2 a^{2}+\mathcal{R}_{x}^{2}+\mathcal{R}_{y}^{2},
$$

and the $\varepsilon_{2}$ for a huge number of events is

$$
\varepsilon_{2}=\frac{\mathcal{R}_{y}^{2}-\mathcal{R}_{x}^{2}}{2 a^{2}+\mathcal{R}_{x}^{2}+\mathcal{R}_{y}^{2}} .
$$

The initial eccentricity decreases with increasing $a$, and the effect of the droplet radius $a$ on $\varepsilon_{2}$ becomes slight when $\mathcal{R}_{x}$ and $\mathcal{R}_{y}$ are large. These conclusions are consistent with the results in Fig. 3, where $\sigma_{0} \sim a$, the values of elliptic flow decrease with increasing $\sigma_{0}$ for the RHIC sources, and the values of elliptic flow for the LHC sources are almost independent of $\sigma_{0}$ because the LHC sources have larger initial source sizes. In Eqs. (10) and (11), $N_{d}$, 


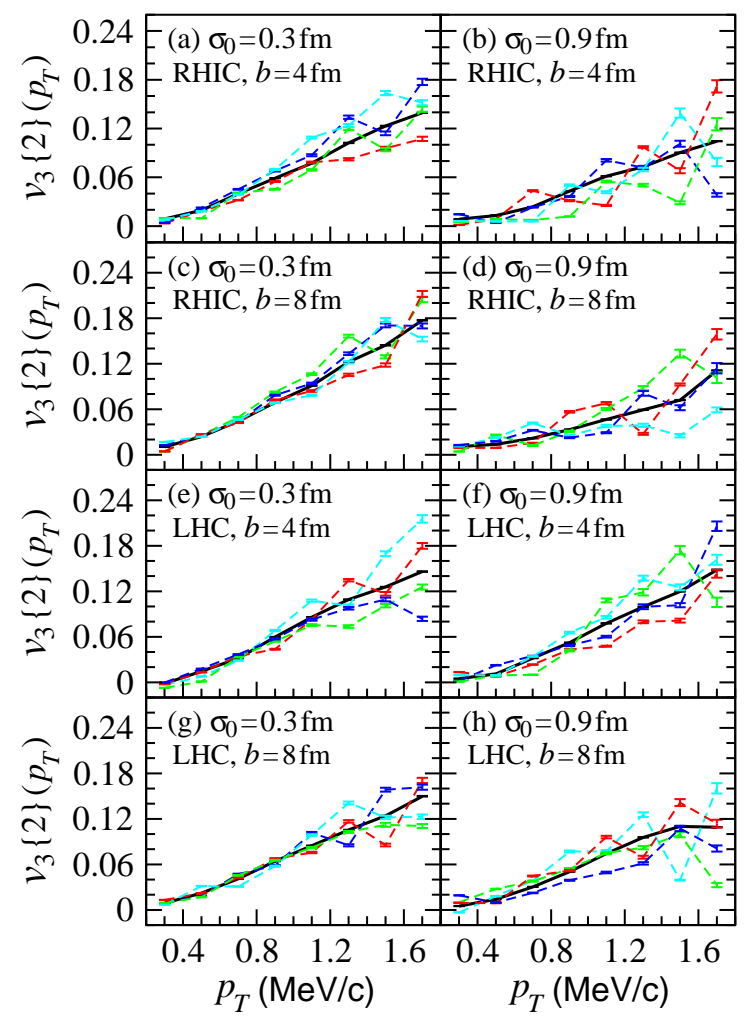

FIG. 5: (Color online) The pion triangular flow of the event subcollections (dashed lines) as in Fig. 4. The solid lines are the average results over sixty the subcollections.

$X_{i}$, and $Y_{i}$ vary chaotically event to event. It leads to the fluctuations of the elliptic flow of single event and the event subcollections with finite number of events as shown in Fig. 4.

In Fig. 5 the dashed lines show the triangular flow for the event subcollections as in Fig. 4. The solid lines are the results averaged over sixty the subcollections. As compared to the results of elliptic flow in Fig. 4, the fluctuations of triangular flow are larger. The values of triangular flow are related to the initial eccentricity $\varepsilon_{3}$, which becomes nonzero due to the granular inhomogeneous structure of the initial sources. Because involving elliptic integrations, we cannot obtain an analytic expression of $\varepsilon_{3}$ even for the simple droplet model.

In the third and sixth columns of Table I, we list the values of $\varepsilon_{2}$ and $\varepsilon_{3}$ calculated in Eq. (8) by averaging with the initial distributions of energy density (see the top panels in Fig. 1) of the six thousand events for the RHIC and LHC sources with the different $b$ and $\sigma_{0}$ values, respectively. The values of $\varepsilon_{2}$ and $\varepsilon_{3}$ increase with increasing $b$ and decrease with increasing $\sigma_{0}$ for fixed $b$. The columns 4 and 7 of Table I list the average values of $v_{2}\{2\}\left(p_{T}\right)$ and $v_{3}\{2\}\left(p_{T}\right)$ over $p_{T}$ and sixty the subcollections each of them with 100 events. 
TABLE I: The values of $\sigma_{0}$ (column 2), eccentricities (columns 3 and 6), average flow harmonics columns 4 and 7), scaled average flow harmonics (columns 5 and 8), average transverse diameter (columns 9), and granularity length (column 10) of the initial sources for the RHIC and LHC collisions with impact parameters $b=4$ and $8 \mathrm{fm}$.

\begin{tabular}{c|ccccccccc}
\hline \hline & $\sigma_{0}(\mathrm{fm})$ & $\varepsilon_{2}$ & $\left\langle v_{2}\{2\}\left(p_{T}\right)\right\rangle$ & $\widetilde{v}_{2}$ & $\varepsilon_{3}$ & $\left\langle v_{3}\{2\}\left(p_{T}\right)\right\rangle$ & $\widetilde{v}_{3}$ & $2\{\rho\}(\mathrm{fm})$ & $L_{\xi}(\mathrm{fm})$ \\
\hline RHIC & 0.3 & 0.189 & 0.082 & 0.434 & 0.091 & 0.071 & 0.780 & 6.57 & 6.80 \\
$b=4 \mathrm{fm}$ & 0.6 & 0.183 & 0.072 & 0.393 & 0.085 & 0.061 & 0.718 & 6.68 & 7.67 \\
& 0.9 & 0.172 & 0.065 & 0.378 & 0.076 & 0.052 & 0.684 & 6.87 & 9.47 \\
\hline RHIC & 0.3 & 0.375 & 0.137 & 0.365 & 0.143 & 0.085 & 0.594 & 5.20 & 5.49 \\
$b=8 \mathrm{fm}$ & 0.6 & 0.356 & 0.126 & 0.351 & 0.129 & 0.062 & 0.480 & 5.37 & 6.71 \\
& 0.9 & 0.322 & 0.117 & 0.363 & 0.110 & 0.045 & 0.409 & 5.62 & 9.53 \\
\hline LHC & 0.3 & 0.172 & 0.063 & 0.366 & 0.076 & 0.071 & 0.934 & 7.30 & 7.50 \\
$b=4 \mathrm{fm}$ & 0.6 & 0.167 & 0.062 & 0.371 & 0.075 & 0.071 & 0.947 & 7.40 & 8.27 \\
& 0.9 & 0.165 & 0.062 & 0.376 & 0.065 & 0.068 & 1.046 & 7.58 & 9.79 \\
\hline LHC & 0.3 & 0.367 & 0.131 & 0.357 & 0.112 & 0.075 & 0.670 & 5.81 & 6.08 \\
$b=8 \mathrm{fm}$ & 0.6 & 0.350 & 0.130 & 0.371 & 0.105 & 0.071 & 0.676 & 5.97 & 7.11 \\
& 0.9 & 0.325 & 0.125 & 0.385 & 0.092 & 0.061 & 0.663 & 6.19 & 9.35 \\
\hline \hline
\end{tabular}

For fixed $b$, the variations of $\left\langle v_{2}\{2\}\left(p_{T}\right)\right\rangle$ and $\left\langle v_{3}\{2\}\left(p_{T}\right)\right\rangle$ with $\sigma_{0}$ are consistent with the variations of $\varepsilon_{2}$ and $\varepsilon_{3}$ with $\sigma_{0}$, respectively. However, the values of $\left\langle v_{3}\{2\}\left(p_{T}\right)\right\rangle$ and $\varepsilon_{3}$ for the same $\sigma_{0}$ but different $b$ are not completely consistent. For instance, the value of $\varepsilon_{3}$ for the RHIC source with $\sigma_{0}=0.9 \mathrm{fm}$ and $b=4 \mathrm{fm}$ is 0.076 and smaller than the value 0.110 for the RHIC source with the same $\sigma_{0}$ and $b=8 \mathrm{fm}$, but the corresponding $\left\langle v_{3}\{2\}\left(p_{T}\right)\right\rangle$ value for the former source is 0.052 and larger than the $\left\langle v_{3}\{2\}\left(p_{T}\right)\right\rangle$ value 0.045 for the later source. This contradiction of the values of $\left\langle v_{3}\{2\}\left(p_{T}\right)\right\rangle$ and $\varepsilon_{3}$ for different $b$ indicates that there exist other effects on the triangular flow values for different impact parameter. In the fifth and eighth columns of Table I, we present the ratios $\widetilde{v}_{2} \equiv\left\langle v_{2}\{2\}\left(p_{T}\right)\right\rangle / \varepsilon_{2}$ and $\widetilde{v}_{3} \equiv\left\langle v_{3}\{2\}\left(p_{T}\right)\right\rangle / \varepsilon_{3}$, respectively. One can see that the values of the scaled average elliptic flow and triangular flow, $\widetilde{v}_{2}$ and $\widetilde{v}_{3}$, are insensitive to $\sigma_{0}$. 


\section{B. Fluctuations of the pion flow harmonics and HBT correlation functions of event} subcollections

From Figs. 4 and 5 it can be seen that the elliptic flow and triangular flow of the event subcollections (dashed lines) are fluctuated. These fluctuations are smoothed out in the observables for all the event subcollections (solid lines). To display the flow fluctuations of the event subcollections in the analysis for all events (or all event subcollections), we introduce the distribution $d N / d f$ of the differences,

$$
f_{v n}=\left|v_{n}^{(i)}\{2\}\left(p_{T}\right)-v_{n}^{(j)}\{2\}\left(p_{T}\right)\right|, \quad(j \neq i),
$$

accumulating for all the event subcollections and the $p_{T}$ bins in the considered region. Here, the superscript denotes the event subcollection.

Unlike some cumulate quantities which smooth out the fluctuations in the analysis for all events, $d N / d f$ is a fluctuation distribution. It becomes wide for the variables $v_{n}^{(i)}\{2\}$ with large fluctuations, and the similar analysis was used in the investigations of the fluctuations of single-event HBT correlation functions [14, 16, 28]. Because the event number of collision is very huge (in principle it may reach any large number if prolonging experiment time), the number of the event subcollections will be large enough to overcome the influence of statistic fluctuations on the distributions. In Fig. 6, we plot the distributions $d N / d f_{v n}$ for the RHIC and LHC sources with different impact parameter $b$ and $\sigma_{0}$ values. The widths of the distributions increase with increasing $\sigma_{0}$, and in most case the widths decrease with increasing $b$ for fixed $\sigma_{0}$. The distributions of $d N / d f_{v 3}$ are wider as compared to the corresponding $d N / d f_{v 2}$ distributions, and the width of the distribution of triangular flow is sensitive to $\sigma_{0}$.

The wider the distributions $d N / d f_{v n}$, the larger the fluctuations are. The width of the distribution reflects the fluctuation magnitude of the flow harmonics. The changes of the widths of the distributions $d N / d f_{v 2}$ and $d N / d f_{v 3}$ with impact parameter $b$ and $\sigma_{0}$ reflect the variations of the fluctuation magnitudes with the granular inhomogeneity of the initial sources. In order to quantify the granular inhomogeneity of the initial source, we introduce a granularity length of the initial source as the product of the initial transverse radius $\mathcal{R}_{\perp}$ and the transverse granularity parameter $\xi_{\perp}$ [43] of the initial source,

$$
L_{\xi}=\mathcal{R}_{\perp} \xi_{\perp}=\mathcal{R}_{\perp} \frac{\left(\mathcal{R}_{\perp} / \sigma_{0}\right)^{2}}{N_{d}-2} \approx \frac{2\{\rho\}^{3}}{\{\rho\}^{2}-4 \sigma_{0}^{2}}, \quad\left(2 \sigma_{0}<\{\rho\}\right) .
$$




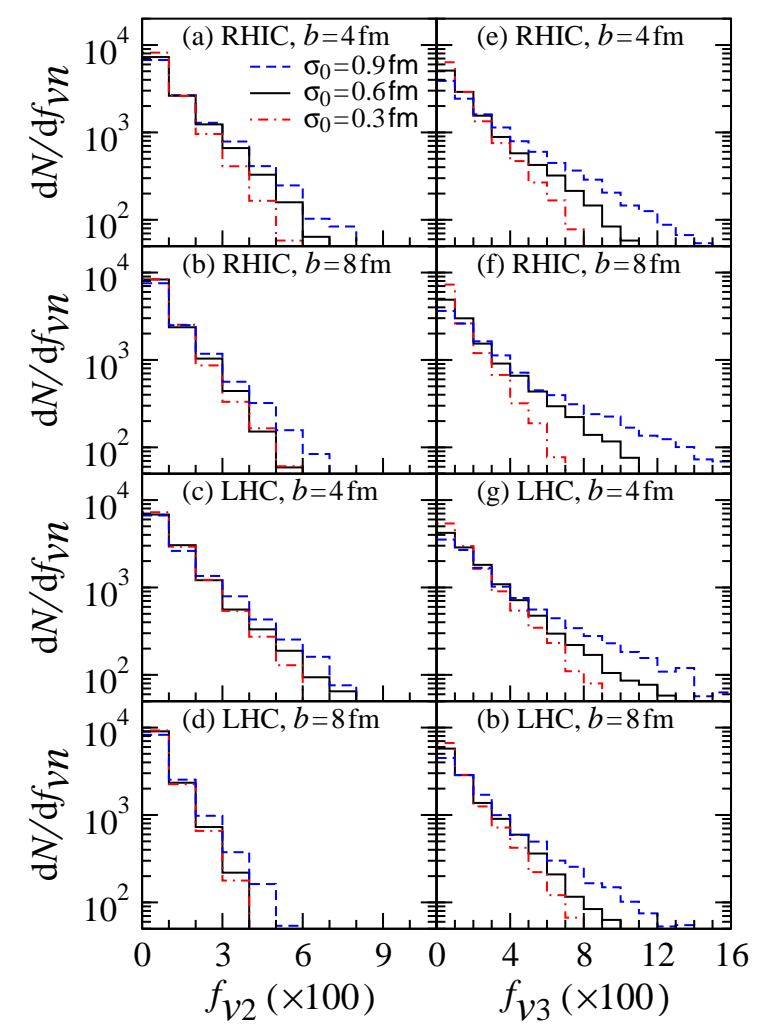

FIG. 6: (Color online) The distributions $d N / d f_{v n}$ for the RHIC and the LHC sources with different impact parameter $b$ and $\sigma_{0}$.

Here, we replace $\mathcal{R}_{\perp}$ approximately with the average transverse radius of the initial source $\{\rho\}$, and replace $N_{d}$ approximately with $\frac{1}{2}\left(\{\rho\} / \sigma_{0}\right)^{2}$, considering approximately the same numbers of the hot spot and cold valley in the initial source. Clearly, the concept of granular source requires $N_{d} \geq 2$. So we have $2\{\rho\}<L_{\xi}<\infty$. From Eq. (15), the granularity length increase when the initial source radius $\{\rho\}$ (associated with collision impact parameter and energy) increases. For fixed $\{\rho\}, L_{\xi}$ increases with increasing $\sigma_{0}$ because the droplet number decreases with increasing $\sigma_{0}$.

In the right two columns of Table I, we presents the values of $2\{\rho\}$ (calculated with the initial energy density of source) and $L_{\xi}$ for the RHIC and the LHC sources respectively. The values of $\{\rho\}$ decrease with increasing impact parameter $b$ and increase slightly with increasing $\sigma_{0}$ for fixed $b$. The values of $\{\rho\}$ for the LHC sources are larger than the corresponding results for the RHIC sources. In most case, the value of $L_{\xi}$ for smaller $b$ is larger than that for larger $b$ with the same $\sigma_{0}$, because the initial source radius is larger for smaller b. However, for $\sigma_{0}=0.9 \mathrm{fm}$, the $L_{\xi}$ value for the RHIC source with $b=8 \mathrm{fm}$ is larger 
than that for $b=4 \mathrm{fm}$. This is because that the effect of droplet number on the granularity length becomes important when $\left(\{\rho\} / \sigma_{0}\right)^{2}$ is small.

From Fig. 6 and the $L_{\xi}$ values in Table I, we observe that the widths of the distributions $d N / d f_{v 2}$ and $d N / d f_{v 3}$ become wider as $L_{\xi}$ increases, for the RHIC sources or the LHC sources. This is obvious for the triangular flow distributions. We conclude that the fluctuation magnitude of the triangular flow of event subcollections is sensitively dependent on the granularity length of the initial source. For the sources of the same collision energy, the fluctuation magnitude of the triangular flow increases with $L_{\xi}$ monotonically. However, for different collision energies, we observe the variation of the distribution width with $L_{\xi}$ is not completely in a monotonic manner. For instance, the $L_{\xi}$ values for the LHC source with $b=8 \mathrm{fm}$ and $\sigma_{0}=0.6 \mathrm{fm}$ is $7.11 \mathrm{fm}$ and larger than the $L_{\xi}$ value $6.71 \mathrm{fm}$ for the RHIC source with the same $b$ and $\sigma_{0}$, but the distribution width for the LHC source is smaller than that for the RHIC source. This indicates the limitation of $L_{\xi}$ for the collisions with greatly different energies.

For granular sources, the single- or several-event HBT correlation functions are fluctuated [14, 16, 27, 28]. We plot in Fig. 7 the two-pion HBT correlation functions of the event subcollections (dashed lines) each of them with 10 the events for the RHIC and the LHC sources with different impact parameters and $\sigma_{0}=0.6 \mathrm{fm}$. Here, $q_{\text {side }}$ and $q_{\text {out }}$ are the relative transverse momenta of the pion pair in the "side" and "out" directions [44, 45]. The solid lines are the results averaged over six-hundred the subcollections. We observe the fluctuations of the HBT correlations of the event subcollections, and the fluctuations are smoothed out in the average results. As the analyses for the flow fluctuations, we investigate the distribution, $d N / d f_{C q}$, for the HBT correlation functions $C\left(q_{\text {side }}\right)$ and $C\left(q_{\text {out }}\right)$. In Fig. 8, we plot the distributions of $d N / d f_{C q}$ the RHIC and the LHC sources with different $b$ and $\sigma_{0}$ values. We observe that the distributions are insensitive to $\sigma_{0}$, although they become wider as compared to the distributions for the source with smoothed initial conditions [14, 16]. Unlike elliptic flow and triangular flow, HBT correlation functions reflect more about the source freeze-out geometry and dynamics rather than the initial details of the sources.

The width of the distribution $d N / d f$, thus the fluctuation magnitude, can be described by the root-mean-square (RMS), $f_{\text {rms }}$, quantitatively. Finally, we plot in Fig. 9 the variations of the RMS of the elliptic flow, triangular flow, and HBT correlation functions with the granularity length of the initial source. One can see that the RMS values of the flow 


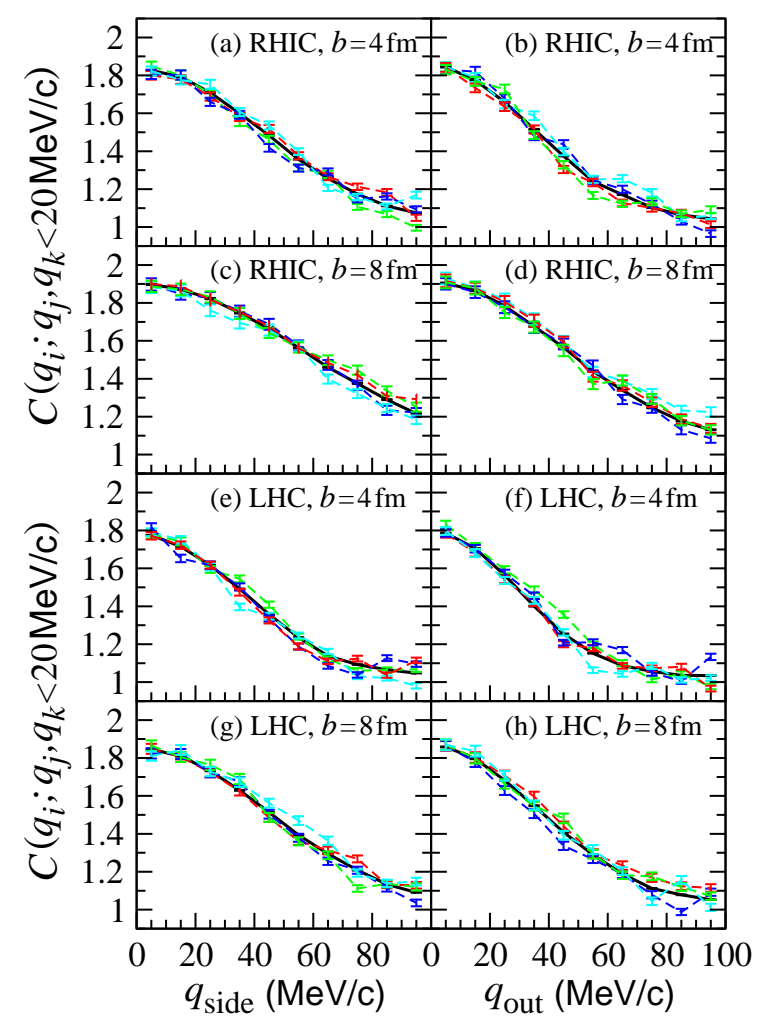

FIG. 7: (Color online) The HBT correlation functions of the event subcollections (dashed lines) each of them with the 10 events for the RHIC and the LHC sources with different impact parameters and $\sigma_{0}=0.6 \mathrm{fm}$. For each event the particle pair number is $10^{6}$. The solid lines are the average results over six hundred the subcollections.

harmonics increase as $L_{\xi}$ increases. But the RMS values of the HBT correlation functions are almost independent of $L_{\xi}$. The RMS values of the triangular flow are very sensitive to $L_{\xi}$. This dependence provide a way to study the granular inhomogeneity of the initial sources through the analyses of the fluctuations of triangular flow in ultrarelativistic heavy ion collisions.

\section{SUMMARY AND DISCUSSIONS}

We investigate the fluctuations of pion elliptic flow, triangular flow, and HBT correlation functions for the hydrodynamic sources of the Au-Au collisions at $\sqrt{s_{N N}}=200 \mathrm{GeV}$ and the $\mathrm{Pb}-\mathrm{Pb}$ collisions at $\sqrt{s_{N N}}=2.76 \mathrm{TeV}$. The initial sources are generated by the HIJING, and then evolve described by ideal relativistic hydrodynamics in $(2+1)$ dimensions with 


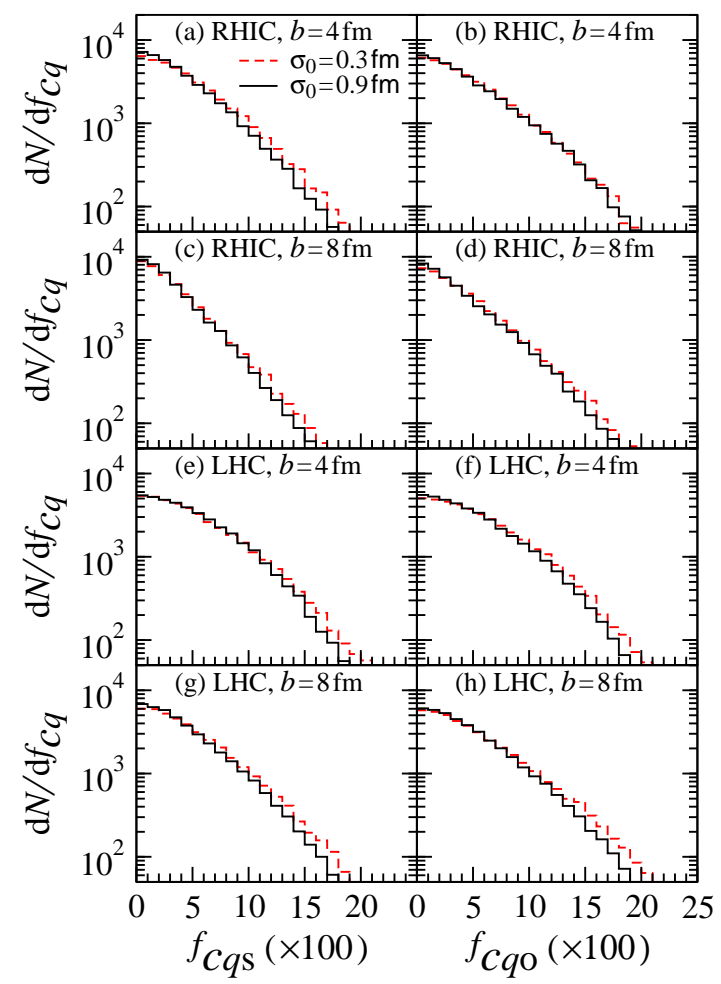

FIG. 8: (Color online) The distributions $d N / d f_{C q}$ for the RHIC and the LHC sources with different values of impact parameter $b$ and $\sigma_{0}$.
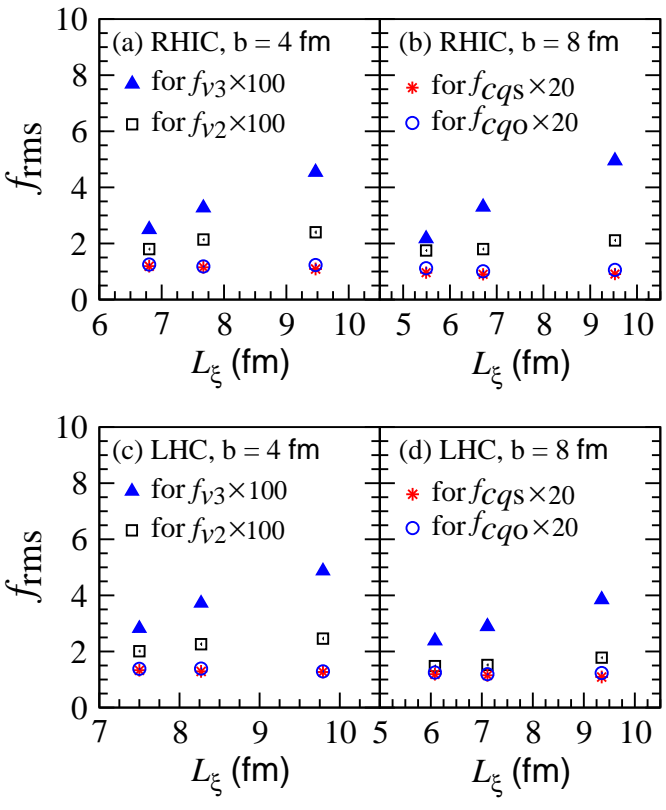

FIG. 9: (Color online) The variations of the root-mean-square $f_{\text {rms }}$ of the elliptic flow, triangular flow, and HBT correlation functions with $L_{\xi}$, for the RHIC and LHC sources with $b=4$ and $8 \mathrm{fm}$. 
Bjorken's longitudinal boost invariance. The EOS of s95p-PCE, which combines the hadron resonance gas at low temperature and the lattice QCD results at high temperature, is employed in the hydrodynamic calculations. For the hydrodynamic sources with the FIC, the elliptic flow, triangular flow, and HBT correlation functions are fluctuated event-by-event. These FIC-caused fluctuations survive in the observables obtained with event subcollections. To display the fluctuations we introduce the fluctuation distribution, $d N / d f$, of the observable of event subcollections, which becomes wide for the observable with large fluctuations. We also introduce the granularity length $L_{\xi}$ to describe the granular inhomogeneity of the initial sources. The relationships between the granularity length and the fluctuations of the pion elliptic flow, triangular flow, and HBT correlation functions of event subcollections are investigated. Our investigations indicate that the FIC lead to event-by-event fluctuations of the elliptic flow, triangular flow, and HBT correlation functions. These FIC-caused fluctuations can be detected by the fluctuation distributions of the observables of event subcollections. The fluctuations of the triangular flow of event subcollections are sensitive to the granularity length of the initial source. This dependence provide a way to investigate the granular inhomogeneity of the initial sources through analysing the fluctuations of triangular flow in ultrarelativistic heavy ion collisions.

For the initial source with fluctuating matter distribution, the initial velocities of fluidcells in the source is usually nonzero and also fluctuated. Considering the very low ratio of shear viscosity to entropy density of the QGP matter and the stability of the hydrodynamic evolution of the FIC sources with nonzero initial velocities of fluid-cells in the source, we use an ideal hydrodynamics model in this work. On the other hand, we also did not consider the initial fluctuation in longitudinal direction in this work. Further investigations on the relationship between the source initial granular inhomogeneity and the fluctuations of final observables for different initial source models and based on viscous hydrodynamic description of source evolution will be of great interest.

\section{Acknowledgments}

We thank Dr. Longgang Pang and Dr. Luan Cheng for helpful discussions. This work is supported by the National Natural Science Foundation of China under Contract No. 
11275037.

[1] For a review see, A. Adare, M. Luzum and H. Petersen, Summary of the major findings and discussions of the workshop "Initial State Fluctuations and Final State Correlations", held at ECT* in Trento in July, 2012, arXiv:1212.5388; Phys. Scripta 87 (2013) 048001; M. Luzum and H. Petersen, J Phys. G 41 (2014) 063102.

[2] B. Schenke, P. Tribedy, and R. Venugopalan, Phys. Rev. Lett. 108 (2012) 252301; ibid., Phys Rev. C 86 (2012) 034908.

[3] B. Alver, G. Roland, Phys. Rev. C 81 (2010) 054905.

[4] B. Alver, C. Gombeaud, M. Luzum, J. Y. Ollitrault, Phys. Rev. C 82 (2010) 034913.

[5] P. Staig, E. Shuryak, Phys. Rev. C 84 (2011) 034908.

[6] B. Schenke, S. Jeon, C. Gale, Phys. Rev. Lett. 106 (2011) 042301; ibid., Phys. Rev. C 85 (2012) 024901; ibid., Phys. Lett. B 702 (2011) 59.

[7] K. Aamodt et al. (ALICE Collaboration), Phys. Rev. Lett. 107 (2011) 032301; A. Adare et al. (PHENIX Collaboration), Phys. Rev. Lett. 107 (2011) 252301; G. Ada et al. (ATLAS Collaboration), Phys. Rev. C 86 (2012) 014907; CMS Collaboration, JHEP 02 (2014) 088.

[8] C. Gale, S. Jeon, B. Schenke, P. Tribedy, R. Venugopalan, Phys. Rev. Lett. 110 (2013) 012302.

[9] L. X. Han, G. L. Ma, Y. G. Ma, et al., Phys. Rev. C 84 (2011) 064907.

[10] L. Ma, G. L. Ma, Y. G. Ma, Phys. Rev. C 89 (2014) 044907.

[11] L. V. Bravina, B. H. Brusheim, G. Kh. Eyyubova, V. L. Korotkikh it et al., Eur. Phys. J. C 74 (2014) 2807.

[12] M. Gyulassy, D. H. Rischke, B. Zhang, Nucl. Phys. 613 (1997) 397.

[13] T. Osada, C.E. Aguiar, Y. Hama, and T. Kodama, in Proc. of the 6th RANP Workshop, Eds. T. Kodama et al., World Scientific, Singapore (2001), P. 174 nucl-th/0102011.

[14] Y. Y. Ren, W. N. Zhang, J. L. Liu, Phys. Lett. B 669 (2008) 317.

[15] K. Werner, Iu. Karpenko, T. Pierog, M. Bleicher, and K. Mikhailov, Phys. Rev. C 82 (2010) 044904 .

[16] Hu Ying, Su Zhongqian, Zhang Weining, Nuclear Science and Techniques 24 (2013) 050522.

[17] X. N. Wang, M. Gyulassy, Phys. Rev. D 44 (1991) 3501; M. Gyulassy, X. N. Wang, Comp. Phys. Commun. 83 (1994) 307. 
[18] J. D. Bjorken, Phys. Rev. D 27 (1983) 140.

[19] C. Shen, U. Heinz, P. Huovinen, H. C. Song, Phys. Rev. C 82 (2010) 054904.

[20] D. H. Rischke, arXiv:nucl-th/9809044

[21] A. Harten, P. D. Lax. B. van Leer, SIAM Rev., 25 (1983) 35; B. Einfeldt, SIAM J. Numer. Anal., 25 (1988) 294. V. Schneider, U. Katscher, D. H. Rischke et al. J. Comput. Phys. 105 (1993) 92 .

[22] D. H. Rischke, S. Bernard, J. A. Maruhn, Nucl. Phys. A 595 (1995) 346; D. H. Rischke, M. Gyulassy, Nucl. Phys. A 608 (1996) 479.

[23] W. N. Zhang, M. J. Efaaf, C. Y. Wong, M. Khaliliasr, Chin. Phys. Lett. 10 (2004) 1918; M. J. Efaaf, W. N. Zhang, M. Khaliliasr et al., High Energy Phys. \& Nucl. Phys. 29 (2005) 46; ibid. 467.

[24] W. N. Zhang, M. J. Efaaf, C. Y. Wong, Phys. Rev. C 70 (2004) 024903

[25] L. L. Yu, W. N. Zhang, C. Y. Wong, Phys. Rev. C 78 (2008) 014908; H. J. Yin, J. Yang, W. N. Zhang, L. L. Yu, Phys. Rev. C 86 (2012) 024914.

[26] M. J. Efaaf, Z. Q. Su, W. N. Zhang, Chin. Phys. C 36 (2012) 410; arXiv:1008.1531,

[27] C. Y. Wong, W. N. Zhang, Phys. Rev. C 70 (2004) 064904.

[28] W. N. Zhang, Shu-Xia Li, C. Y. Wong, M. J. Efaaf, Phys. Rev. C 71 (2005) 064908.

[29] P. F. Kolb, U. Heinz, arXiv:nucl-th/0305084

[30] G. Baym, B. L. Friman, J. P. Blaizot, M. Soyeur, W. Czyż, Nucl. Phys. A 407 (1983) 397.

[31] Z. W. Lin, C. M. Ko, B. A. Li, B. Zhang, S. Pal, Phys. Rev. C 72 (2005) 064901.

[32] L. G. Pang, Q. Wang, X. N. Wang, Phys. Rev. C 86 (2012) 024911.

[33] G. A. Sod, J. Fluid Mech. 83 (1977) 785.

[34] S. S. Adler et al. (PHENIX Collaboration), Phys. Rev. C 69 (2004) 034909.

[35] R. Preghenella (for ALICE Collaboration), Acta. Phys. Pol. B 43 (2012) 555; arXiv:1111.7080.

[36] J. Adams et al. (STAR Collaboration) Phys. Rev. C 72 (2005) 014904.

[37] S. Voloshin and Y. Zhang, Z. Phys. C 70 (1996) 665.

[38] A. M. Poskanzer and S. A. Voloshin, Phys. Rev. C 58 (1998) 1671.

[39] S. S. Adler et al. (PHENIX Collaboration), Phys. Rev. Lett. 91 (2003) 182301.

[40] S. A. Voloshin, A. M. Poskanzer, R. Snellings, arXiv:0809.2949.

[41] N. Borghini, P. M. Dinh, J. Y. Ollitrault, Phys. Rev. C 64 (2001) 054901.

[42] K. Aamodt et al. (ALICE Collaboration), Phys. Rev. Lett 105 (2010) 252302. 
[43] Z. T. Yang, W. N. Zhang, L. Huo, J. B. Zhang, J. Phys. G. 36 (2009) 015133.

[44] G. Bertsch, M. Gong, M. Tohyama, Phys. Rev. C 37 (1988) 1896; G. Bertsch, Nucl. Phys. A 498 (1989) 173c.

[45] S. Pratt, T. Csörgo, and J. Zimányi, Phys. Rev.C 42 (1990) 2646. 